Journal for ImmunoTherapy of Cancer

\section{antibody in a metastatic bladder cancer associated with novel MSH4 mutation and microsatellite instability}

To cite: Yang Y, Jain RK, Glenn ST, et al. Complete response to anti-PD-L1 antibody in a metastatic bladder cancer associated with novel MSH4 mutation and microsatellite instability. Journal for ImmunoTherapy of Cancer 2020;8:e000128. doi:10.1136/ jitc-2019-000128

- Additional material is published online only. To view please visit the journal online (http://dx.doi.org/10.1136/jitc2019-000128).

YY and RKJ contributed equally.

Accepted 25 February 2020

Check for updates

(C) Author(s) (or their employer(s)) 2020. Re-use permitted under CC BY-NC. No commercial re-use. See rights and permissions. Published by BMJ.

For numbered affiliations see end of article.

Correspondence to Dr Yuanquan Yang; yuanquan.yang@osumc.edu

\section{ABSTRACT}

Background Microsatellite instability (MSI) occurs in $3 \%$ of urothelial carcinomas as a result of germline or somatic loss of function mutation in mismatch repair (MMR) proteins. ${ }^{1}$ Although MSH4 is a member of the DNA MMR mutS family, the association of MSH4 mutation with MSI has not been described. We report a complete responder to PD-L1 blockade who had MSH4 mutated metastatic bladder cancer with mixed histology and MSI. The genomics of urothelial, plasmacytoid and squamous histology was characterized individually through microdissection.

Case presentation An 81-year-old man was diagnosed with metastatic urothelial carcinoma 8 months after a cystectomy for muscle invasive bladder cancer. His disease was primary refractory to first-line platinum-based chemotherapy but attained complete response to secondline atezolizumab. PCR-based assay revealed MSI high. The tumor mutational burden was elevated to 36.7 mut/ $\mathrm{Mb}$. However, immunohistochemistry of MLH1, MSH2, MSH6 and PMS2 was intact. Whole exome sequencing confirmed that the above mentioned four classic MMR genes were wild type but revealed a deleterious MSH4 L359I mutation with variant allele fraction of $30 \%$ and Polyphen2 score of 0.873 . The association of MSH4 alterations and $\mathrm{MSI}-\mathrm{H}$ was independently verified in two publicly available MSI-H colorectal cancer datasets. Conclusions The novel MSH4 L359I mutation is associated with MSI and high mutational burden leading to remarkable response to PD-L1 blockade. More studies are warranted to establish the causality relationship between MSH4 and MSI.

\section{BACKGROUND}

DNA damage repair (DDR) is an important defensive mechanism to maintain genomic stability and avoid tumorigenesis. Alterations in DDR pathways are observed in up to $28 \%$ of high grade urothelial carcinoma (UC) of the bladder (UCB). ${ }^{2}$ The most common mutated genes include ERCC2, ATM and BRCA2 which belong to double-strand DNA break and nucleotide excision repair pathways. DNA mutation in mismatch repair (MMR) defect only occurs in about $3 \%$ of UC, majority of which are upper tract tumors, commonly associated with lynch syndrome. ${ }^{1}$

Classically, the human DNA MMR system is composed of four proteins: MLH1, MSH2, MSH6 and PMS2. The MMR system corrects base-base mispairs introduced into the genome during DNA replication. ${ }^{3}$ MMR defect is as a result of germline or somatic loss of function mutation in MMR proteins. Microsatellites are short, tandemly repeated sequences that occur throughout the genome and are used as markers of defective MMR (dMMR). Tumors with dMMR display highfrequency microsatellite instability (MSI-H). Therefore, dMMR is frequently analyzed by testing for loss of MMR proteins by immunohistochemistry (IHC) or for MSI using a PCRbased assay. ${ }^{4} \mathrm{MSI}-\mathrm{H}$ is a predictive biomarker for response to immune checkpoint inhibitors (ICI). ${ }^{5}$

Although MSH4 is a member of the DNA MMR mutS family, the association of MSH4 mutation with MSI has not been described. ${ }^{6}$ We report a case of metastatic $\mathrm{UCB}$ with mixed histology (urothelial cell carcinoma, plasmacytoid carcinoma and squamous cell carcinoma) and MSI-H which possessed a somatic MSH4 missense mutation and achieved complete response to PD-L1 blockade. We characterized the genomics of each histology separately through microdissection.

\section{CASE REPORT}

An 81-year-old African American man presented with a 2-month history of painless gross hematuria. Medical history included hypertension, sick sinus syndrome and favorable intermediate risk prostate 


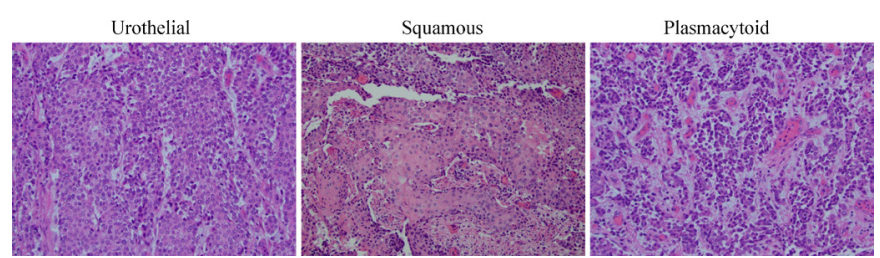

Figure 1 Histological analysis of the primary bladder tumor. Representative images show mixed histology of urothelial, squamous and plasmacytoid variant by H\&E stain (10x).

adenocarcinoma (Gleason grade $3+3=6$, T1c, prostatespecific antigen of $10.5 \mathrm{ng} / \mathrm{ml}$ ) on surveillance. He was an ex-smoker with 10-pack-year smoking history. Other social and family history was unremarkable.

Workup including a cystoscopy followed by a CT urogram revealed a large $5 \mathrm{~cm}$ bladder tumor and several prominent pelvic lymph nodes (up to $1.1 \mathrm{~cm}$ ). He was diagnosed with muscle invasive high-grade UC through transurethral resection of bladder tumor. Staging CT scans did not suggest any distant metastasis. Neoadjuvant cisplatin-based chemotherapy was felt inappropriate due to poor performance status and renal function. He underwent an uncomplicated radical cystoprostatectomy and bilateral extended pelvic lymph node dissection, and ileal conduit urinary diversion. Surgical pathology showed pT2bN0 high-grade UCB with squamous differentiation (15\%) and plasmacytoid features (5\%; figure 1), and pT3bN1 prostate adenocarcinoma (Gleason grade $4+5=9$ ). During the postoperative period, he was followed by surveillance CT and bone scans which showed no evidence of neoplastic process. The PSA nadir was 0.49

Unfortunately, 8 months after cystectomy, he developed metastatic recurrence of a left chest wall mass (measuring $3.8 \times 3.4 \mathrm{~cm}$, figure $2 \mathrm{~A}$ ), the biopsy of which confirmed metastatic UC with extensive tumor necrosis. In addition, there were new CT findings of a left hemipelvic lesion $(3.6 \times 2.8 \mathrm{~cm}$, figure $2 \mathrm{~B})$ consistent with metastasis. He was treated with first-line carboplatin and gemcitabine

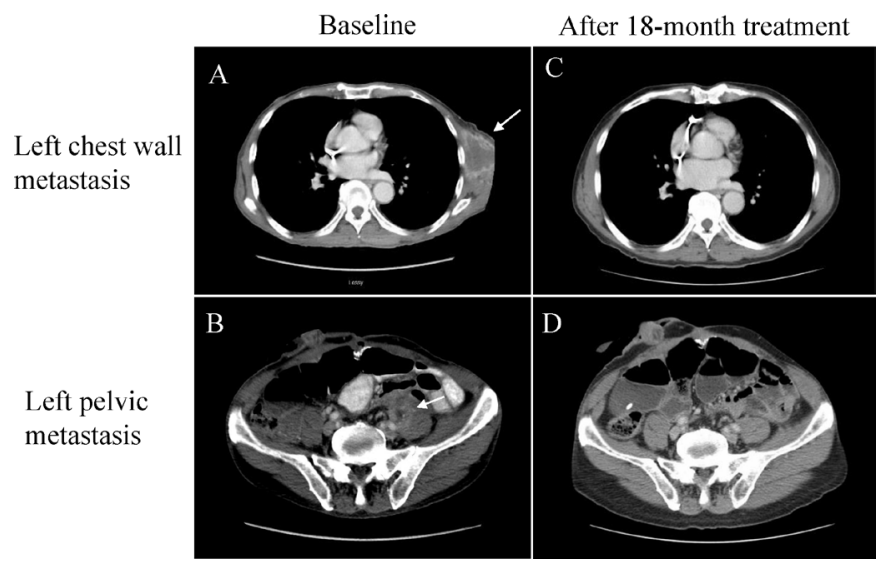

Figure 2 Complete responses to atezolizumab on CT scans. Contrast-enhanced CT scans in cross-sectional views show (A) the baseline left chest wall $(4.7 \times 4.5 \mathrm{~cm})$ and $(B)$ pelvic metastasis $(2.8 \times 2.8 \mathrm{~cm}$ in size, white arrows). Both lesions completely disappeared after 18-month treatment of atezolizumab (C and D, respectively).
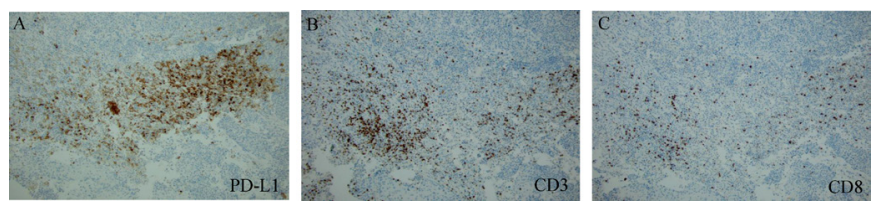

Figure 3 Tumor-infiltrating lymphocytes and PD-L1 staining by immunohistochemistry. Representative images show (A) PD-L1 expression, (B) CD3-positive and (C) CD8-positive Tcell infiltration are enriched in the squamous variant histology. The positive cells are shown in brown (10x).

chemotherapy. However, it was discontinued after three cycles due to disease progression. Afterwards, he received second-line immunotherapy with anti-PD-L1 antibody, atezolizumab. A partial response was observed after cycle 9. A research biopsy of the left chest wall lesion performed after cycle 20 showed only fibrosis with no evidence of disease. He achieved radiographic complete response after 18 months of atezolizumab therapy (figure 2C, D).

\section{Molecular and genetic assessments}

PD-L1 IHC (SP142) of the primary tumor showed overall low positivity $(<5 \%$ of the tumor infiltrating immune cells) but paradoxically increased staining in the squamous histology (figure 3A). CD3-positive and CD8positive tumor-infiltrating lymphocytes (TIL) were also enriched in squamous variant (figure $3 \mathrm{~B}, \mathrm{C}$ ). It suggests that there is histology-dependent heterogeneity of the tumor immune microenvironment (TIME). National Cancer Institute recommended PCR-based MSI testing using five microsatellite markers which revealed MSI-H in all three types of histology. Interestingly, MLH1, MSH2, MSH6 and PMS2 were intact by IHC (data not shown).

In order to investigate the cause of MSI, we performed bulk DNA and RNA-seq of the primary tumor. The whole exome sequencing (WES) confirmed that MLH1, MSH2, MSH6, PMS2 and EPCAM were wild type. However, a somatic missense mutation of MSH4 L359I (1.76288179C>A) was detected. The variant allele frequency was 0.3 . The corresponding amino acid change was located in the core domain MutS III (figure $4 \mathrm{~A}){ }^{7}$ Polyphen2-predicted MSH4 L359I mutation was probably damaging of protein function (score of 0.873).

In addition, two proof reading defects were identified: POLD1 C319R (19.50905983T $>$ C) and POLE R712H (12.133244980C $>$ T) mutations. Polyphen2 suggested that the POLD1 mutation was probably damaging (score of 0.999) and the POLE mutation was benign (score of 0.067). Nevertheless, both mutations were affecting the DNA polymerase domains (figure $4 \mathrm{~B}, \mathrm{C}$ ). The complete list of somatic mutations is listed in online supplementary table S1. There were 2166 altering and truncating mutations in the 58947532 bp capture regions. The calculated tumor mutational burden (TMB) was 36.7 mutations per $\mathrm{Mb}$.

To study the molecular mechanisms of tumor heterogeneity, we performed WES and RNA-seq of each histology after microdissecting the bulk tumor. The mutational 

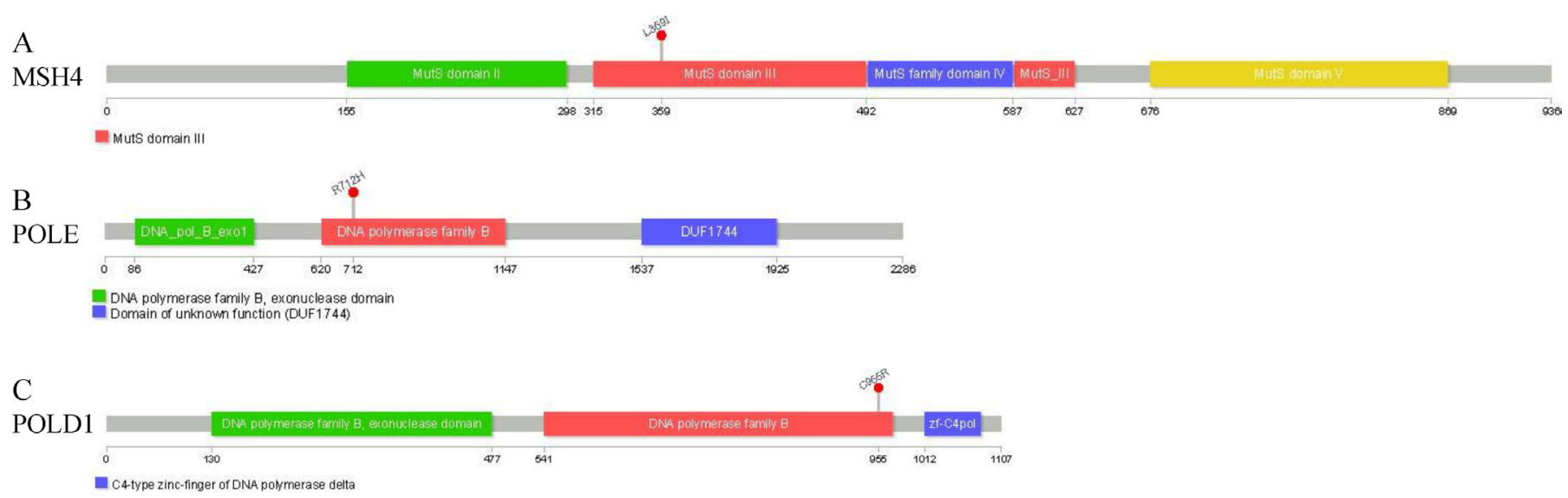

Figure 4 Somatic mutations affecting MSH4, POLE and POLD1. The lollipop plots show the affected loci in the coding proteins by (A) MSH4 L359I, (B) POLE R712H, and (C) POLD1 C319R. The gray bar represents the entire protein with amino acid positions. The colored boxes are specific functional domains. On top of the lollipops, the amino acid change at the specific position is annotated.

landscape in heatmap showed divergent genomic profile of the squamous from urothelial and plasmacytoid variant (online supplementary figure S1). Gene set enrichment analysis demonstrated upregulated chemokine and T-cell receptor signal pathways in the squamous histology (online supplementary figure S2).

\section{DISCUSSION}

MSH4 is one of the seven known MutS homologs. Unlike the other MutS homologs, that recognize mismatched nucleotides to initiate the repair process, MSH4 has not been experimentally implicated in the MMR process and its function is not fully understood. ${ }^{8} \mathrm{MSH} 4$ was first discovered participating in DNA repair during meiosis. ${ }^{6}$ But it was subsequently found to maintain genomic stability in response to ionizing radiation. ${ }^{9}$ To our knowledge, this is the first report of MSI-H associated with a deleterious MSH4 L359I mutation, which is an important addition to the growing body of knowledge in understanding MMR. This conclusion is reached after excluding genetic and epigenetic alteration/silencing of the conventional MMR system by WES and IHC.

To support our conclusion, we next evaluated the mutational status of MSH4 in two independent datasets of MSI-high patients with colorectal cancers from The Cancer Genome Atlas and the Dana Farber Cancer Institute. MSH4 alterations were present in $5 \%$ and $7 \%$ of the MSI-H tumors, respectively. In majority of the cases, the aberrations in MSH4 and the classic MMR genes were mutually exclusive (online supplementary figure S3). Further studies are warranted to establish the causality relationship of MSH4 alteration and MSI.

In addition to dMMR, proofreading defects of POLE and POLD1 were detected in our patient which are increasingly being recognized as another important cause of hypermutators. POLE and POLD1 are subunits of DNA polymerases $\varepsilon$ and $\delta$, respectively, involved in DNA replication and repair. ${ }^{10}$ In addition to DNA polymerase activity, they also possess exonuclease domain, which detects and removes nucleotides. This proofreading function enhances the fidelity of DNA synthesis. The mutation and dysfunction of POLE and POLD1 lead to genome instability and tumorigenesis with high TMB ( $\geq 10 \mathrm{mut} / \mathrm{Mb})$. However, POLE or POLD1 mutants alone do not yield MSI unless there is a concurrent loss of MMR proteins. Approximately $16 \%$ of the MSI tumors have concurrent either a POLE or POLD1 mutation in a pooled analysis. ${ }^{11}$ Only POLE/POLD1 exonuclease mutation in the context of inactivating MMR synergistically increases mutation rate. In our case, the POLD1 C319R and POLE R712H mutations were both affecting the polymerase domains. Therefore, the high TMB is likely due to MMR deficiency rather than proofreading defect.

High TMB an important biomarker for predicting ICI treatment response. ${ }^{12}$ Theoretically, increased mutational load produces higher amount of neoantigens that activate adaptive immune system leading to tumor-specific T-cell proliferation. Using neoantigen prediction pipeline, ${ }^{13}$ we were able to identify 194 tumor-specific neoantigens that had strong MHC-I binding affinity from the DNA-seq data. Of the 194 neoantigens, 93 had confirmed expression by RNA-seq (online supplementary figure S4). The tumor-specific T-cell clonal expansions were uncovered by comparing TCR repertoire of pretreatment and post-treatment peripheral blood (online supplementary figure S5). The specificity of these TCR repertoires to the predicted neoantigens needs to be evaluated in future studies.

The heterogeneity of TIME has been well described. ${ }^{14}$ However, the mechanism is not fully understood. In the present study, the upregulated chemokine and T-cell receptor signal pathways and increased TIL infiltration as well as PD-L1 expression were associated with squamous differentiation. Squamous differentiation is the most common histology variant of UC which occurs in about $20 \%$ of the cases. We investigated the underlying 
molecular mechanism at genomic level by performing WES and RNA-seq of each histology separately. Our data advocate clonal evolution of UC to other histological variants after mutational gains. Among the unique genetic alterations discovered only in squamous variant, NRG1 A14S (8.32579560 .G.T) mutation drew our attention. Physiologically, neuregulin1 (ie, NRG1) is a cell adhesion molecule that interacts with ERBB2 receptor tyrosine kinase to enhance its phosphorylation. ${ }^{15}$ When mutated, NRG1 mutant is associated with increased proinflammatory cytokine release ${ }^{16}$ Phlyphen2 predicted NRG1 A14S probably damaging with score of 0.935 . Taken together, our data suggest that the immunophenotype determined by genotype may contribute to the TIME heterogeneity.

Plasmacytoid urothelial carcinomas (PUC) is a rare histological variant which accounts for $2.9 \%$ of the $\mathrm{UC}^{17}$ The distinctive mutation landscape and diffuse growth pattern are associated with advanced stage and poor response to conventional chemotherapy. ${ }^{18}{ }^{19}$ Alternative treatment strategy is needed to improve outcome. The impact of plasmacytoid variant on treatment response by ICI remains uncertain. The patients with mixed histology are often grouped together in prospective and retrospective studies. ${ }^{20-22}$ In literature review, we found another case report of a patient with metastatic PUC who was treated with first-line pembrolizumab, another ICI, and obtained a partial response. ${ }^{23}$ Thus, our case supports further investigation into first-line ICI therapy for metastatic PUC.

\section{CONCLUSIONS}

Our data are consistent with discovery of MSH4 as a gene whose mutation is associated with MSI-high and increased TMB leading to remarkable response to ICI. MSH4 alterations occurred in 3\% of the patients in the TCGA PanCancer Altas UCB cohort. ${ }^{24}$ We propose the refinement of the MSI screening test (for including MSH4 in the standard IHC panel) and the development of mutated MSH4 as a biomarker for ICI response. The histologydependent heterogeneity of TIME points to preferentially treating mixed histology (bladder) tumors with immunotherapy despite overall low PD-L1 expression.

\section{Author affiliations}

${ }^{1}$ Division of Medical Oncology, The Ohio State University James Cancer Hospital, Columbus, Ohio, USA

${ }^{2}$ Department of Medicine, Roswell Park Cancer Institute, Buffalo, New York, USA ${ }^{3}$ Department of Genitourinary Oncology, Moffitt Cancer Center, Tampa, Florida, USA ${ }^{4}$ Center for Personalized Medicine, Roswell Park Cancer Institute, Buffalo, New York, USA

${ }^{5}$ Department of Pathology, Roswell Park Cancer Institute, Buffalo, New York, USA ${ }^{6}$ Department of Biostatistics and Bioinformatics, Roswell Park Cancer Institute, Buffalo, New York, USA

${ }^{7}$ Center for Immunotherapy, Roswell Park Cancer Institute, Buffalo, New York, USA

Acknowledgements The authors are grateful to the patient for providing permission to share the medical information.

Contributors YY, RJ and SG drafted the manuscript. STG, BX, PKS, LW, QH, ML, NH, JW and SB contributed data.
Funding This work was supported by grants from the National Cancer Institute grant P30CA016056 involving the use of Roswell Park Comprehensive Cancer Center's Pathology Network, Genomics and Bioinformatics Shared Resource, grant U24CA232979, and National Center for Research Resources grant S100D019977.

Competing interests SG reports grants and personal fees from Bristol Myers Squibb, grants and personal fees from Bayer, grants and personal fees from Pfizer, personal fees from Exelixis, grants and personal fees from Janssen, grants and personal fees from Corvus, personal fees from Genentech/Roche, personal fees from Sanofi/ Genzyme, personal fees from EMD Serono, grants from Merck, grants from Immunomedics, grants from Agensys, grants from Novartis, grants from Eisai, grants from Astellas/ Seattle Genetics, grants from Calithera Biosciences, outside the submitted work.

Patient consent for publication Not required.

Ethics approval Ethics approval for a case report is deemed exempt by the Roswell Park IRB.

Provenance and peer review Not commissioned; externally peer reviewed.

Open access This is an open access article distributed in accordance with the Creative Commons Attribution Non Commercial (CC BY-NC 4.0) license, which permits others to distribute, remix, adapt, build upon this work non-commercially, and license their derivative works on different terms, provided the original work is properly cited, appropriate credit is given, any changes made indicated, and the use is non-commercial. See http://creativecommons.org/licenses/by-nc/4.0/

\section{ORCID iD}

Yuanquan Yang http://orcid.org/0000-0002-5209-9247

\section{REFERENCES}

1 lyer G, Audenet F, Middha S, et al. Mismatch repair (MMR) detection in urothelial carcinoma (UC) and correlation with immune checkpoint blockade (ICB) response. J Clin Oncol 2017;35:4511.

2 Dason S, McPherson V, Teo MY, et al. Defining the DNA damage repair (DDR) genomic landscape of urothelial carcinoma of the bladder (UCB). J Clin Oncol 2018;36:502.

3 Kawakami H, Zaanan A, Sinicrope FA. Microsatellite instability testing and its role in the management of colorectal cancer. Curr Treat Options Oncol 2015;16:30.

4 Buhard O, Cattaneo F, Wong YF, et al. Multipopulation analysis of polymorphisms in five mononucleotide repeats used to determine the microsatellite instability status of human tumors. J Clin Oncol 2006;24:241-51.

5 Le DT, Uram JN, Wang $\mathrm{H}$, et al. Pd-1 blockade in tumors with mismatch-repair deficiency. N Engl J Med 2015;372:2509-20.

6 Clark N, Wu X, Her C. Muts homologues hMSH4 and hMSH5: genetic variations, functions, and implications in human diseases. Curr Genomics 2013;14:81-90.

7 Obmolova G, Ban C, Hsieh P, et al. Crystal structures of mismatch repair protein MutS and its complex with a substrate DNA. Nature 2000;407:703-10.

8 Ross-Macdonald P, Roeder GS. Mutation of a meiosis-specific MutS homolog decreases crossing over but not mismatch correction. Cell 1994;79:1069-80.

9 Chu Y-L, Wu X, Xu Y, et al. Muts homologue hMSH4: interaction with elF3f and a role in NHEJ-mediated DSB repair. Mol Cancer 2013;12:51.

10 Park VS, Pursell ZF. Pole proofreading defects: contributions to mutagenesis and cancer. DNA Repair 2019;76:50-9.

11 Rayner E, van Gool IC, Palles C, et al. A panoply of errors: polymerase proofreading domain mutations in cancer. Nat Rev Cancer 2016;16:71-81.

12 Chan TA, Yarchoan M, Jaffee E, et al. Development of tumor mutation burden as an immunotherapy biomarker: utility for the oncology clinic. Ann Oncol 2019;30:44-56.

13 Yadav M, Jhunjhunwala S, Phung QT, et al. Predicting immunogenic tumour mutations by combining mass spectrometry and exome sequencing. Nature 2014;515:572-6.

14 Jiménez-Sánchez A, Memon D, Pourpe S, et al. Heterogeneous Tumor-Immune microenvironments among differentially growing metastases in an ovarian cancer patient. Cell 2017;170:927-38.

15 Britsch S. The neuregulin-I/ErbB signaling system in development and disease. Adv Anat Embryol Cell Biol 2007;190:1-65.

16 Marballi K, Quinones MP, Jimenez F, et al. In vivo and in vitro genetic evidence of involvement of neuregulin 1 in immune system dysregulation. J Mol Med 2010;88:1133-41. 
17 Ericson KJ, Thomas L, Lee BH. Plasmacytoid variant urothelial carcinoma: clinicopathologic outcomes and experience with neoadjuvant chemotherapy. J Clin Oncol 2019;37:483.

18 Al-Ahmadie HA, lyer $\mathrm{G}$, Lee $\mathrm{BH}$, et al. Frequent somatic $\mathrm{CDH} 1$ lossof-function mutations in plasmacytoid variant bladder cancer. Nat Genet 2016;48:356-8.

19 Teo MY, Al-Ahmadie H, Seier K, et al. Pre-Operative chemotherapy (CTX) in plasmacytoid urothelial carcinoma (puc). J Clin Oncol 2018;36:522.

20 Bellmunt J, de Wit R, Vaughn DJ, et al. Pembrolizumab as secondline therapy for advanced urothelial carcinoma. N Engl J Med 2017;376:1015-26.

21 Powles T, Durán I, van der Heijden MS, et al. Atezolizumab versus chemotherapy in patients with platinum-treated locally advanced or metastatic urothelial carcinoma (IMvigor211): a multicentre, open-label, phase 3 randomised controlled trial. Lancet 2018;391:748-57.

22 Agarwal A, Nassar A, Pond GR, et al. Impact of pure versus mixed metastatic urothelial carcinoma (mUC) histology on response with immune checkpoint inhibitors (ICls). J Clin Oncol 2019;37:479.

23 Hunter L, Moser J, Sturge C, et al. First-Line pembrolizumab therapy in a cisplatin-ineligible patient with plasmacytoid urothelial carcinoma: a case report. $J$ Oncol Pharm Pract 2019;1078155219835006.

24 Cerami E, Gao J, Dogrusoz U, et al. The cBio cancer genomics portal: an open platform for exploring multidimensional cancer genomics data. Cancer Discov 2012;2:401-4. 